\title{
Application of Network-Constrained Transactive Control to Electric Vehicle Charging for Secure Grid Operation.
}

Hu, Junjie; Yang, Guangya; Bindner, Henrik W.; Xue, Yusheng

Published in:

IEEE Transactions on Sustainable Energy

Link to article, DOI:

10.1109/TSTE.2016.2608840

Publication date:

2016

Document Version

Peer reviewed version

Link back to DTU Orbit

Citation (APA):

Hu, J., Yang, G., Bindner, H. W., \& Xue, Y. (2016). Application of Network-Constrained Transactive Control to Electric Vehicle Charging for Secure Grid Operation. IEEE Transactions on Sustainable Energy, 8(2), 505 - 515. https://doi.org/10.1109/TSTE.2016.2608840

\section{General rights}

Copyright and moral rights for the publications made accessible in the public portal are retained by the authors and/or other copyright owners and it is a condition of accessing publications that users recognise and abide by the legal requirements associated with these rights.

- Users may download and print one copy of any publication from the public portal for the purpose of private study or research.

- You may not further distribute the material or use it for any profit-making activity or commercial gain

- You may freely distribute the URL identifying the publication in the public portal 


\title{
Application of Network-Constrained Transactive Control to Electric Vehicle Charging for Secure Grid Operation
}

\author{
Junjie Hu, Member, IEEE, Guangya Yang, Senior Member, IEEE, Henrik W. Bindner, Member, IEEE, \\ and Yusheng Xue, Member, IEEE
}

\begin{abstract}
This paper develops a network-constrained transactive control method to integrate distributed energy resources (DERs) into a power distribution system with the purpose of optimizing the operational cost of DERs and power losses of the distribution network as well as preventing grid problems including power transformer congestion and voltage violations. In this method, a price coordinator is introduced to facilitate the interaction between the distribution system operator and aggregators in the smart grid. Electric vehicles are used to illustrate the proposed network-constrained transactive control method. Mathematical models are presented to describe the operation of the control method. Finally, simulations are presented to show the effectiveness of the proposed method. To guarantee its optimality, we also checked the numerical results obtained with the network-constrained transactive control method and compared them with the one solved by centralized control, and found a good performance of the proposed control method.
\end{abstract}

Index Terms-Distributed decision making, grid-interactive energy sources, network-constrained operation, transactive control.

$\mathbf{T}$

\section{INTRODUCTION} photovoltaic generation, electric vehicles etc flexible loads requires enhanced operation at distribution system level as well as closer interaction between distribution system level operation and transmission system level operation. For example, as suggested in [1], the functions at distribution system level should include grid operator function and market operator function. The grid operator secures the network operation while the market operator coordinates the electricity purchase and sale, and the interchange of power to other markets. In [2], a hierarchical electric market structure consisting of wholesale electricity market and distribution network electricity market is proposed to facilitate the coordination of energy markets in distribution and transmission networks. The proposed market structure

Manuscript received January 25, 2016; revised May 14, 2016 and August 10, 2016; accepted September 7, 2016. Date of publication; date of current version. This work is supported by the Danish iPower project funded by the Danish Agency for Research and Innovation under Grant 0603-00435B. Paper no. TSTE-00077-2016.

J. Hu, G. Yang, and H. Bindner are with the Center for Electric Power and Energy, Department of Electrical Engineering, Technical University of Denmark, Kgs. Lyngby 2800, Denmark (e-mail: junhu@elektro.dtu.dk; gyy@elektro. dtu.dk; hwbi@elektro.dtu.dk).

Y. Xue is with the State Grid Electric Power Research Institute, Nanjing 210003, China (e-mail: xueyusheng@sgepri.sgcc.com.cn).

Digital Object Identifier 10.1109/TSTE.2016.2608840 enables the integration of microgrids, which provide energy and ancillary services in distribution networks.

The enhanced operation at distribution system level makes it possible to explore and engage DERs' flexibility potentials via different approaches, centralized mechanism have been proposed in studies [3], [4]. In [3], the proposed system integrates demand side management and active distributed generation in the wholesale market via an centrally optimized EMS (energy management system), which allows a better exploitation of renewable energy sources and a reduction of the customers energy consumption costs with both economic and environmental benefits. To distinguish the characteristics of inflexible load and flexible load, the authors in [4] presented optimal pricing tariff for flexible loads in distribution networks which ensures cost saving for them. The optimal pricing tariff is solved centrally by an load serving entity sitting at distribution system level. Although the centralized approach yields the optimal outcome from the global perspective, the method has drawbacks in term of its communication and computational scalability, privacy concerns issue. Alternatively, transactive control is proposed and promoted to manage the operation of DERs resources and flexibilities. Transactive control is defined as "a set of economic and control mechanisms that allows the dynamic balance of supply and demand across the entire electrical infrastructure using value as a key operational parameter" by the GridWise Architecture Council [5] and has been successfully applied in several demonstration projects in the US and Europe [6]. The intent of the control framework is to reach equilibriums by standardizing a scalable, distributed mechanism via exchanging information about generation, consumptions, constraints and responsive assets over dynamic, real-time forecasting periods using economic incentive signaling, and thus solving the increasingly complex power system problems.

In [7], a transactive control method named "PowerMatcher" was developed to balance supply and demand in electricity networks. In the PowerMatcher method each device is represented by a control agent, which tries to operate the process associated with the device in an economically optimal way. The design of the PowerMatcher is based on the theoretical finding that computational economies of local control agents using a dynamic pricing mechanism are able to handle scarce resources adaptively in ways that are optimal locally as well as globally. In [8], a hierarchical transactive control architecture is proposed to integrate renewables in smart grids considering the operation at primary, secondary and tertiary control levels. The transactive 
control framework is applied at the tertiary control level with the purpose of using optimal allocation of resources in the presence of uncertainties in terms of renewables and loads. In [9], an integrated dynamic market mechanism is proposed that combines real-time market and frequency regulation allowing renewable generators and flexible consumers to iteratively negotiate electricity prices, with purpose of reducing the cost of regulation reserves. In [10], a transactive control framework is used to coordinate a population of thermostatically controlled loads with the purpose of allocating energy economically subject to a peak energy constraint. A mechanism is proposed in the paper to implement the desired social choice function in dominant strategy equilibrium.

As transactive control's application to electric vehicle (EV) integration studies, the authors in [11] propose a scalable threestep approach to manage the charging of electric vehicles on the demand side with the purpose of minimizing charging cost of EVs. The three steps consist of aggregation, optimization and control. Transactive control is applied in the third step, i.e., the real-time control step to divide the optimal power generated in step 2 among the individual EVs, which is determined by a priority-based scheme. The work is further developed in [12] where an event-driven dual coordination mechanism is presented at the real-time control level. The simulation result indicated that the number of messages exchanged with the EVs was significantly reduced, by at least $64 \%$.

Although the transactive control framework has been widely used in the smart grid to reach an energy balance between supply and demand as well as for demand response management [7]-[12], such studies do not consider the network that is an indispensable factor in operational study. For example, as indicated in [13]-[15], a large penetration of EVs also means new loads on the electric utilities, and undesirable congestion and voltage violations may exist in the distribution network when the batteries are recharged because of uncoordinated or solely cost-minimization-based charging. The latter means the EVs react to the wholesale price/regulating power price in a correlated way, for example, all EVs are charged when electricity prices are low, it might create a new peak demand at that time. Typically, the challenges in the distribution grid caused by the increasing electricity consumption of EVs are resolved by expensive expansion of the grid to match the size and the pattern of demand. Alternatively, in a smart grid context, the problem of violation of grid constraints can also be solved smartly using advanced control strategies such as transactive control supported by an increased use of information and communication technology. To address the conflicting challenges, transactive control frameworks were used in [16] for the charging of electric vehicles that incorporated distribution transformer and voltage constraints. A hierarchical multi-agent structure was used in [16] that consists of auctioneer agent, substation agent, and EV device agent. The substation agent summed up the bid functions of all the underlying EV device agents in a low voltage network and in turn sent the bid function to the unique auctioneer agent who defined the equilibrium price. In addition, the substation agent also ensured that the grid constraints were not violated given the possible equilibrium price. But, the current application of transactive control [7]-[12], [16] mainly focuses on real time operation that may limit its application in power systems where "scheduling and control" is a vital and useful operational principle.

This paper develops a multiple periods network-constrained transactive control method to integrate distributed energy resources (DERs) into the power distribution system, in particular using electric vehicles as an illustration. By the term network-constrained transactive control, we mean that network constraints including power transformer capacity and voltage limitations are considered in transactive control applications for integrating distributed energy resources like electric vehicles. With the extension to multiple periods, the energy intertemporal characteristics of DERs, such as the dynamics of EV charging can be considered in the optimization. To implement the proposed network-constrained transactive control, a price coordinator is introduced in this study to coordinate the power flow between the distribution network operator and commercial actors, i.e., the aggregators, which fits the operations under the deregulated electricity market environment. As a result of including network constraints, the method will be able to provide granular information for locational marginal prices of each period at each bus. Besides, the method also includes power loss in the objective function that is one of the concerns of distribution operation. In addition, we compare the optimality of the numerical result obtained with the network-constrained transactive control method with one solved by centralized control; the results indicate good performance of the proposed transactive control method.

The remainder of the paper is organized as follows. In Section II, an energy management system using a transactive control framework is described to integrate distributed energy resources. A network-constrained transactive control method is presented in Section III. Section IV presents simulations to illustrate the performance of the proposed method. Finally, discussion and conclusions are made in Section V.

\section{CONTROL SySTEM DESCRIPTION}

Fig. 1 presents the network-constrained transactive control system for distributed energy resources integration. In the system, several aggregators are specified to manage DERs and interact with a distribution system operator and a price coordinator to eliminate grid congestion and prevent voltage violations. The current system specifically introduces a price coordinator that facilitates the interactions between the DSO and aggregators. Note that the energy dispatch used is based on the spot market, since the aggregators procure the electricity when the price is low. The state of the distribution network is not considered which means a conflicting situation might happen, e.g., aggregators who aim to procure the energy from the spot market in a lower price period, while the power brings operational challenges to distribution networks.

In order to integrate DERs smoothly into the distribution network, novel control relationships are needed for the management system. In the proposed two-stage control system: 1) each aggregator centrally generates an individually optimal energy 


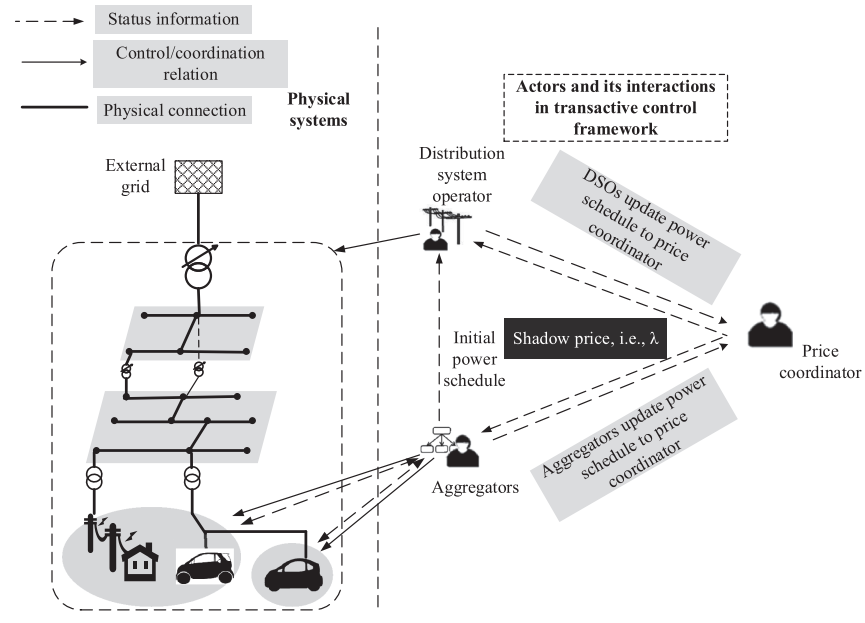

Fig. 1. A network-constrained transactive control system for distributed energy resources integration.

schedule for DERs as well as an aggregated power schedule over the whole scheduling period; 2 ) the aggregators and DSO interact with the price coordinator to reach a power consensus on each bus of the distribution network via iterative information exchange on price and power, if the aggregators' power schedule could potentially cause network problems to DSO. The information exchange on the power schedule and the shadow price i.e. $\lambda(i, l)$ used by the transactive control can be enabled and operated by the DSO, the aggregators and the price coordinator based on current infrastructure. Note regarding how to handle the shadow price in practice, suggestions have been made in the literature. In [16], the authors assumed that the customers are not charged the equilibrium price in the auction-based market/transactive control, instead, the equilibrium price is interpreted as a control signal that guarantees the necessary reserves are provided. Alternatively, it is argued in [5] that dynamic price at distribution system level should have real economical incentive. We recognise the value of $\lambda(i, l)$ represents a compromise between the utility of customer and the interests of grid, which shares similar features of the distribution locational marginal prices in [17]. Although straight-forward and easy to implement, the model [17] brings about the risk of causing new peaks in the grid due to unconfirmed power schedule of aggregators to the DSO. Instead, the method proposed in this study can guarantee explicit power limits issued to the aggregators for the DSO when solving grid congestion, because the price and the power schedules are fixed after a price-clearing mechanism. Furthermore, the implementation of the shadow price in the settlement phase is out of the scope of the paper but will be addressed in the future work from the authors.

Key operations of the three actors in the system are presented as follows:

1) Aggregator's role and operational functions: Aggregators provide energy services to DER users and coordinate with the DSO and price coordinator. Note the role of the aggregator here is similar to a retailer who on-behalf of customers to buy the electricity in the energy spot market. To support such a role, two stages are needed: DER energy schedule generation and interaction with the DSO and price coordinator. In the first stage, aggregators collect information from the users to make an optimal energy schedule for DERs. Then, this initial energy schedule will be shared with the DSO to form the baseline. The baseline is normally defined as an estimate of the electricity that would have been consumed by a customer in the absence of a demand response event [18]. This implies that if there are no potential network problems, the aggregators' initial schedule will be accepted by the DSO; otherwise, this baseline will be used for later on cost function formulation.

2) DSO's role and operational functions: To ensure secure operation of the distribution network, the non-profit organization DSO needs to interact with the aggregators and price coordinator, exchanging buses' information on the network with the aggregators and the price coordinator and responding to the price set by price coordinator. Besides, DSO is informed about aggregators' initial power schedule since it will keep tracking the power schedule when responding to the price set by the price coordinator.

3) Price coordinator's role and operational functions: The price coordinator is an authorized entity to determine the shadow prices and facilitates the interactions between the DSO and the aggregators to reach a power consensus at each bus of the network. The price coordination center could be operated by a third party. The proposed third party is feasible ${ }^{1}$ if more distributed energy resources are connected on the distribution network level. The independent third party could be used to provide such services to different distribution system operators and aggregators, for example, in Denmark, there are around 70 distribution companies which serves electricity to publics. In addition, the proposed third party could ensure fairness to aggregators and DSOs. If the price coordinator is operated by a DSO, it may discriminate some aggregators if their operational schedules have conflicts with DSO's own interests. From our view, the price coordinator should be a non-profit organization but will charge certain operational fee to its customers including DSOs and aggregators to maintain its operation and development.

\section{Mathematical Modeling OF NeTWORK-CONSTRAined TRANSACTIVE CONTROL}

In this section, mathematical models of the networkconstrained transactive control method are introduced. An electric vehicle is used as an example to illustrate the developed transactive control method. Fig. 2 shows the functions and interactions of the entities in the proposed model. We start with the aggregator who uses linear programming to formulate an aggregated EV charging schedule in Stage I. The charging

${ }^{1} \mathrm{http}: / /$ www.ipower-net.dk/news. In the Danish iPower smart grid project, a flexibility clearing house software infrastructure is developed that enables Distribution System Operators and aggregators to interact, so the potential flexibility controlled by the aggregators can be provided to the DSOs in a market-based way. 


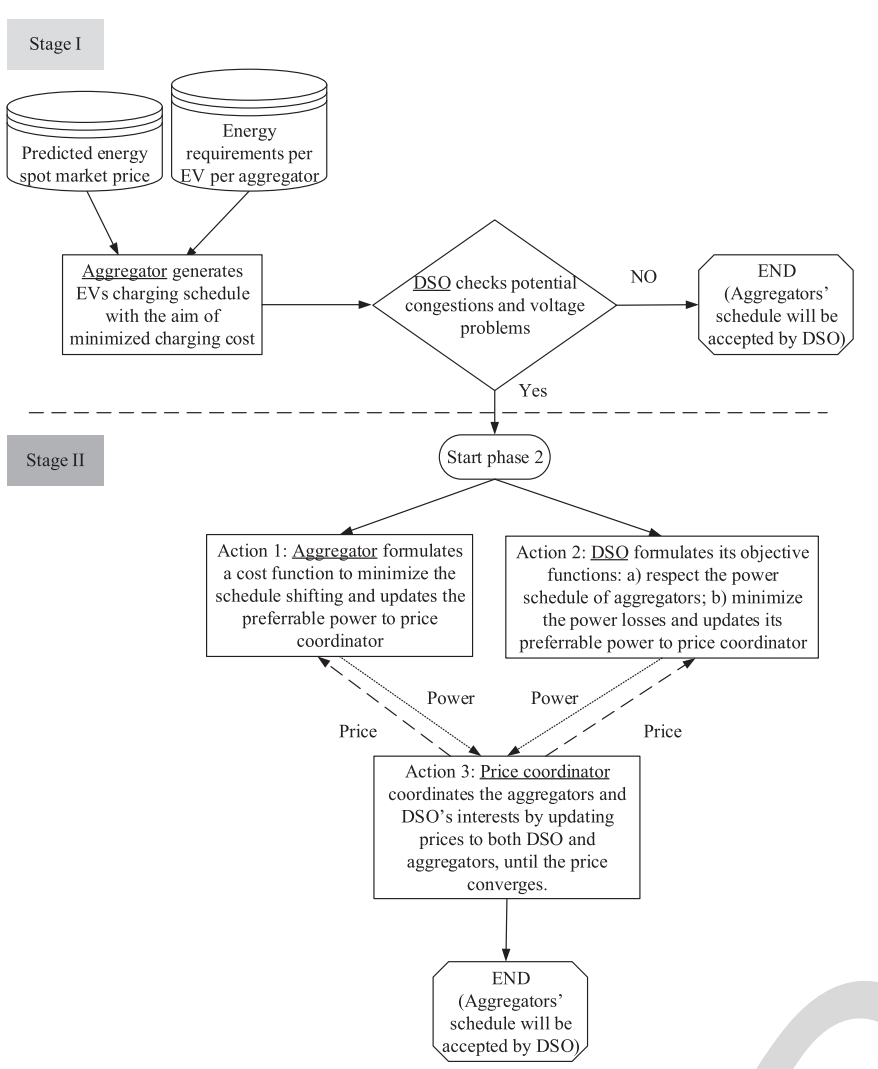

Fig. 2. Flowchart of the proposed method that describes the function and interactions of entities.

schedule forms a baseline of the flexibility cost function used in section III-B where the modeling development of the networkconstrained transactive control is presented in Stage II. Finally, a distributed computational algorithm is presented in Stage II that facilitates implementation of the transactive control.

\section{A. Stage I: Aggregator's Electric Vehicles Charging}

\section{Schedule Generation}

A linear programming-based electric vehicle charging optimization is formulated and used by the aggregators to generate the optimal charging schedule, assuming knowledge of EV users' driving pattern and forecast electricity spot price. Note that the linear programming model and the assumptions adopted here may not accurately characterize the charging process of the electric vehicles in terms of the uncertainty of EV users' driving pattern, battery charging behavior, EV charging efficiency etc., however, as discussed in [19], it is a sufficient method for generating the optimal charging schedule to minimize the charging cost.

The charging objective is to minimize the charging cost as well as to fulfill the individual EV's energy requirements for the next twenty-four hours, and the discharging ability and battery degradation cost are not considered in the study. The solution is introduced similarly for each aggregator:

$$
\min \sum_{j=1}^{N_{k}^{E}} \sum_{i=1}^{N_{T}} \Phi_{j, i} P_{j, i} t,
$$

subject to

$$
\left\{\begin{array}{l}
S O C_{0, j} \cdot E_{\mathrm{cap}, j}+\sum_{i=1}^{N_{T}} P_{j, i} t_{j, i}=S O C_{\mathrm{Max}, j} \cdot E_{\mathrm{cap}, j} \\
0 \leq P_{j, i} \leq P_{\mathrm{max}, j}, i=1, \ldots, N_{T}
\end{array}\right.
$$

where

$P_{j, i} \quad$ Optimization variable, the $j^{\text {th }} \mathrm{EV}$ charging power

$N_{k}^{E} \quad$ Number of EVs under aggregator $k$. at time interval $i$.

$N_{T} \quad$ Number of time slots in the scheduling period.

$j \quad$ Index for the number of EVs under each aggregator, $j=1,2, \ldots, N_{k}^{E}$.

$i \quad$ Index of time slot in the scheduling period, $i=$ $1,2, \ldots, N_{T}$.

311

$\Phi_{j, i} \quad$ Predicted day-ahead electricity market price vector.

$t \quad$ Length of each time slot.

$S O C_{0, j} \quad$ Initial SOC of individual EV.

$S O C_{\mathrm{Max}, j}$ Requested/targeted maximum SOC of individual $\mathrm{EV}$ at the end of the charging period.

$P_{\max , j} \quad$ Maximum charging rate of individual EV.

$E_{\text {cap }, j} \quad$ Capacity of the battery of the EV.

In (1), the first constraint means that the energy to be charged should be equal to the requested energy at the end of the charging period for each electric vehicle. The second constraint represents that the charging rate is less than or equal to its maximum power rate of a charger. The physical meaning of the optimization variable vector $P_{j, i}$ is to make a decision on the charging power in the planned time slots, where the charging cost can be minimized.

With the above optimization problem, the aggregator can generate a unique energy schedule for individual $\mathrm{EV}$ as well as an aggregated power schedule in each time slot. Note that, when interacting with the DSO, the aggregator needs to provide charging locations of the aggregated charging schedules, which is assumed to be known by the aggregators. The previously obtained $P_{j, i}$ will be denoted as $P_{j, i, l} . l$ is the bus index of the distribution network, $l=1, \ldots, N_{B}$. Thus, we calculate the sum of the individual EV energy schedule inside one aggregator $k$ at bus $l$ in time slot $i$ and the total power is denoted as $P_{k, i, l}^{E}$, and

$$
P_{k, i, l}^{E}=\sum_{j \mapsto l} P_{j, i, l}, k=1, \ldots, N_{F}, i=1, \ldots, N_{T}, l=1, \ldots, N_{B}
$$

where

$j \mapsto l$ The electric vehicles of each aggregator connected at 346 bus $l$.

$N_{F} \quad$ Number of aggregators.

$N_{B} \quad$ Number of buses.

$k \quad$ Index for the number of aggregators, $k=1, \ldots, N_{F}$.

$P_{k, i, l}^{E} \quad$ Power requirements of EVs of aggregator $k$ in time slot 351 $i$ at bus $l$.

Note that the EV model used here does not consider the un- 353 certainty of the EV travel pattern, thus the aggregated power 354 consumption of the aggregator might deviate from the planned 355 schedule which will certain influence the accuracy of this model. 356 
This problem can be mitigated by: 1) when the size of the aggregator is bigger such as many flexible resources are controlled by the aggregator, since the uncertainty of individual EV can be evened, and 2) an agreement could be made between the aggregator and the customers that communicate timely on the customers' next day traveling plan.

\section{B. Stage II: Network-Constrained Transactive Control Modeling}

In this study, the principle for applying the networkconstrained transactive control application is that the DSO needs to check whether the charging schedule of aggregators will result in network operation violations. If there is a violation, a congestion price will be generated by the price coordinator to reflect the violations. Otherwise, the power schedule of aggregators will be accepted by the DSO.

To start the modeling of the control method, we propose a flexibility cost function that represents the cost of the power preference difference of aggregators in each time slot $i$ per bus $l$,

$$
\mu_{k}=\zeta_{k}\left(\tilde{P}_{k, i, l}\right)
$$

To facilitate the understanding, we assume

$$
\mu_{k}=C_{k, i, l}\left(\tilde{P}_{k, i, l}-P_{k, i, l}^{E}\right)^{2},
$$

subject to

$$
\sum_{i=1}^{N_{T}} \tilde{P}_{k, i, l} \cdot t_{i}=\sum_{j \mapsto l}\left(S O C_{\mathrm{Max}, j}-S O C_{0, j}\right) \cdot E_{\mathrm{cap}, j}
$$

where $k, i, l$ remain the same with the above notation, $\tilde{P}_{k, i, l}$ denotes the optimization variable, $P_{k, i, l}^{E}$ is the optimized power schedule shown in (2), $C_{k, i, l}$ means the weighting factor which are associated with the power difference, the larger $C_{k, i, l}$ means smaller difference preferred since the objective is to reduce the power shifting. The constraint in (3) means the individual EV energy requirements should always be fulfilled. The flexibility cost function $\mu_{k}$ intends to penalize the deviation from its originally optimized schedule $P_{k, i, l}^{E}$

For the DSO, the objective is to track and regulate the power schedule from aggregators with respect to the operational constraints such as the transformer thermal capacity and the voltage limitations and to minimize the network losses:

$$
\min a \cdot \sum_{i=1}^{N_{T}} \sum_{l=1}^{N_{B}}\left(P_{\text {trans }}(i, l)-\sum_{k=1}^{n_{F}} P_{k, i, l}^{E}\right)^{2}+b \cdot P_{\text {loss }}
$$

$1 \quad$ subject to

$$
\begin{gathered}
\sum_{l=1^{N_{B}}} P_{\mathrm{trans}}(i, l) \leq P_{\mathrm{trans}}^{\mathrm{Max}}(i), \\
U_{0}(i, l)+\Delta U(i, l) \geq U_{\mathrm{Min}}(i, l)
\end{gathered}
$$

where

$a, b \quad$ Weighting factors.

$P_{0} \quad$ Conventional load profiles.
$P_{\text {trans }}(i, l) \quad$ Optimization variable and its physical meaning is the desirable power of DSO for EVs charging, exclude the base load profile.

$n_{F} \quad$ Number of aggregators which has EVs attached in bus $l$.

$A \quad$ Full bus incidence matrix, $N_{B} \times N_{\text {Line }}$, associated to the reference direction of branches. If bus $m$ is the initial node of branch $[m, n], A(m, n)=1$, else $A(m, n)=-1$. Note the matrix is not necessary a square matrix.

$N_{\text {Line }} \quad$ Number of branches.

395 396 397 398 399 400 401 402 403 404 $P_{\text {trans }}^{\operatorname{Max}}$

Power transformer capacity for all the aggregators, for example, it can be estimated by the DSO after deducting the conventional loads.

$U_{0}(i, l)$ The initial voltage of the buses of the network.

$U_{\mathrm{Min}}(i, l) \quad$ The minimum allowable voltage of the buses of the network.

Note that normally in practice, the non-profit organization DSO aims to ensure the safe and efficient operation of the network, provide non-discriminate electricity distribution services to customers, and minimize energy losses of the system. In this study, we proposed that the DSO also aims to supply the desired power schedule of aggregators as much as possible, in addition to the loss minimization objective. It is envisioned in the near future smart grid, the DSO can adapt the objective functions like the one presented in (4) with the real needs.

In (4),

$$
\begin{gathered}
P_{\text {loss }}=\sum_{i=1}^{N_{T}} \sum_{l=1}^{N_{B}}\left(\frac{P_{\text {line }}^{2}(i, l)+Q_{\text {line }}^{2}(i, l)}{V^{2}}\right) R_{l} \\
P_{\text {line }}(i, l)=\left(A \cdot A^{T}\right)^{-1} \cdot A \cdot\left(P_{0}(i, l)+P_{\text {trans }}(i, l)\right)
\end{gathered}
$$

where $P_{\text {loss }}=\sum_{i=1}^{N_{T}} \sum_{l=1}^{N_{B}}\left(\frac{P_{\text {line }, l}^{2}(i)+Q_{\text {line }, l}^{2}(i)}{V^{2}}\right) R_{l}$ can be ap- 422 proximated as $P_{\text {loss }}=\sum_{i=1}^{N_{T}} \sum_{l=1}^{N_{B}} P_{\text {line }}^{2}(i, l) R_{l}$, since Q is usu- 423 ally small in low voltage network, and as long as the voltage 424 is close to nominal. $\Delta U(i, l)$ is calculated from the following 425 simplified equation [20], [21]

$$
\left[\begin{array}{c}
\Delta P \\
\Delta Q
\end{array}\right]=\left[\begin{array}{ll}
\frac{\partial P}{\partial \Theta} & \frac{\partial P}{\partial U} \\
\frac{\partial Q}{\partial \Theta} & \frac{\partial Q}{\partial U}
\end{array}\right]\left[\begin{array}{c}
\Delta \Theta \\
\Delta U
\end{array}\right]
$$

Denote $\mathrm{J}$ the load flow Jacobian from the last iteration,

$$
J=\left[\begin{array}{ll}
\frac{\partial P}{\partial \Theta} & \frac{\partial P}{\partial U} \\
\frac{\partial Q}{\partial \Theta} & \frac{\partial Q}{\partial U}
\end{array}\right]
$$

then the voltage increment can be calculated by the injection 428 increment times the reverse of the Jacobian, as shown below, 429

$$
\left[\begin{array}{c}
\Delta \Theta(i, l) \\
\Delta U(i, l)
\end{array}\right]=J^{-1}\left[\begin{array}{c}
\Delta P(i, l) \\
\Delta Q(i, l)
\end{array}\right]=J^{-1}\left[\begin{array}{c}
P_{\text {trans }}(i, l) \\
0
\end{array}\right]
$$

Here, we assume the reactive power injection increment is zero. 430 $\Theta$ means voltage angle and it is not considered in the study. 431 
Thus we have

$$
\Delta U(i, l)=J_{\mathbf{2 1}}^{-1} \cdot P_{\text {trans }}(i, l) .
$$

433

434 where $J_{21}^{-1}$ means only a submatrix of $J^{-1}$ is used.

From a social fairness point of view, it is desirable to minimize the cost to the aggregator as well as minimizing the power losses and mitigating the impact on the distribution system operator. The social welfare maximization is mathematically formulated as follows:

$$
\begin{aligned}
& \min \sum_{k=1}^{N_{F}} \sum_{i=1}^{N_{T}} \sum_{l=1}^{N_{B}} C_{k, i, l}\left(\tilde{P}_{k, i, l}-P_{k, i, l}^{E}\right)^{2} \\
& \quad+a \cdot \sum_{i=1}^{N_{T}} \sum_{l=1}^{N_{B}}\left(P_{\text {trans }}(i, l)-\sum_{k=1}^{n_{F}} P_{k, i, l}^{E}\right)^{2}+b \cdot P_{\text {loss }}
\end{aligned}
$$

subject to

$$
\begin{aligned}
\sum_{k=1}^{n_{F}} \tilde{P}_{k, i, l} & =P_{\text {trans }}(i, l), i=1, \ldots, N_{T}, \\
\sum_{i=1}^{N_{T}} \tilde{P}_{k, i, l} \cdot t_{i} & =\sum_{j=1}^{N_{k}^{E}}\left(S O C_{\mathrm{cap}, j}-S O C_{0, j}\right) \cdot E_{\mathrm{cap}, j}, \\
\sum_{l=1}^{N_{B}} P_{\text {trans }}(i, l) & \leq P_{\text {trans }}^{\mathrm{Max}}(i), \\
U_{0}+\Delta U & \geq U_{\mathrm{Min}},
\end{aligned}
$$

where the optimization variables of this optimization problem are $\tilde{P}_{k, i, l}$ and $P_{\text {trans }}(i, l)$. The first constraint of (7) implies that sum of the new optimal power of aggregators should be equal to the new optimal power of the DSO. Let $\lambda(i, l)$ denote the Lagrange multiplier corresponding to the first constraint of (7), and keep the rest of the constraints implicit, so the Lagrangian function for (7) is

$$
\begin{aligned}
L\left(\lambda(i, l), \tilde{P}_{k, i, l}, P_{\text {trans }}(i, l)\right)= & \\
& \sum_{k=1}^{N_{F}} \sum_{i=1}^{N_{T}} \sum_{l=1}^{N_{B}} C_{k, i, l}\left(\tilde{P}_{k, i, l}-P_{k, i, l}^{E}\right)^{2} \\
& +a \cdot \sum_{i=1}^{N_{T}} \sum_{l=1}^{N_{B}}\left(P_{\text {trans }}(i, l)-\sum_{k=1}^{n_{F}} P_{k, i, l}^{E}\right)^{2}+b \cdot P_{\text {loss }} \\
& +\sum_{i=1}^{N_{T}} \sum_{l=1}^{N_{B}} \lambda(i, l) \cdot\left(\sum_{k=1}^{n_{F}} \tilde{P}_{k, l, i}-P_{\text {trans }}(i, l)\right)
\end{aligned}
$$

where the optimization variables of optimization problem (8) are $\lambda(i, l), \tilde{P}_{k, i, l}$ and $P_{\text {trans }}(i, l)$.

\section{Stage II: Network-Constrained Transactive Control Implementation}

In order to solve the optimization problem (8), this section applies a distributed computing algorithm which has been applied in several studies [22], [23]. The Lagrangian minimization can be solved by subgradient methods [24] which usually require multiple iterations or information exchange. In the iter- 455 ation, the minimization problems are seen to be decomposable 456 to the DSO and to the aggregators. Specifically, the subgradient 457 method consists of the following iterations, indexed by $\omega$ and 458 initialized with arbitrary $\lambda_{1}^{*}(i, l) \geq 0$ :

1) aggregator minimization at step $\omega$

$$
\begin{gathered}
\min \left(\sum_{k=1}^{N_{F}} \sum_{i=1}^{N_{T}} \sum_{l=1}^{N_{B}} C_{k, i, l}\left(\tilde{P}_{k, i, l}-P_{k, i, l}^{E}\right)^{2}+\right. \\
\left.\sum_{i=1}^{N_{T}} \sum_{l=1}^{N_{B}} \lambda_{\omega}^{*}(i, l) \sum_{k=1}^{n_{F}} \tilde{P}_{k, i, l}\right) \\
\text { s.t. } \sum_{i=1}^{N_{T}} \tilde{P}_{k, i, l} \cdot t_{i}=\sum_{j \in l}\left(S O C_{\text {cap }, j}-S O C_{0, j}\right) \cdot E_{\text {cap }, j}
\end{gathered}
$$

To solve problem (9) and obtain the value of optimization vari- 461 able $\tilde{P}_{k, i, l}$ we use CVX, a package for specifying and solving 462 convex programs [25], [26].

2) DSO minimization at step $\omega$

$$
\begin{array}{r}
\min a \cdot \sum_{i=1}^{N_{T}} \sum_{l=1}^{N_{B}}\left(P_{\text {trans }}(i, l)-\sum_{k=1}^{n_{F}} P_{k, i, l}^{E}\right)^{2}+ \\
b \cdot P_{\text {loss }}-\sum_{i=1}^{N_{T}} \sum_{l=1}^{N_{B}} \lambda_{\omega}^{*}(i, l) P_{\text {trans }}(i, l)
\end{array}
$$

$$
\begin{array}{ll}
\text { s.t. } & \sum_{l=1}^{N_{B}} P_{\text {trans }}(i, l) \leq P_{\text {trans }}^{\mathrm{Max}}(i), \\
& U_{0}(i, l)+\Delta U(i, l) \geq U_{\mathrm{Min}}(i, l)
\end{array}
$$

To solve problem (10) and get the value of optimization vari- 465 able $P_{\text {trans }}(i, l)$, we use CVX and MATPOWER, a MATLAB 466 power system simulation package.

3) Price coordinator: lagrangian multiplier updating for step $\omega+1$

$$
\lambda_{\omega+1}(i, l)=\lambda_{\omega}^{*}(i, l)+\alpha_{\omega} \cdot\left(\sum_{k \in l} \tilde{P}_{k, i, l}^{*}-P_{\text {trans }}(i, l)^{*}\right)
$$

where $\omega$ is the index for the iterations, $\tilde{P}_{k, l, i}^{*}$ is the solution of 470 problem (9), $P_{\text {trans }}(i, l)^{*}$ is the solution of (10), $\alpha_{\omega} \in R$ denotes 471 the step size and can be chosen as $\alpha_{\omega}=\alpha$ which is a positive 472 constant and with the choice, the convergence is guaranteed 473 [24]. Note that $\lambda$ is converged at each bus in each time slot. A 474 simple step size is chosen here to update the $\lambda$, but as discussed 475 in [24], some heuristic approaches can be performed to improve 476 the convergence speed.

\section{CAse Study}

\section{A. Case Specification}

1) EV charging parameters: Two EV penetration levels are 480 studied, i.e., the $50 \% \mathrm{EV}$ level and the $100 \% \mathrm{EV}$ level. All the 481 EVs are affiliated to either aggregator 1 (Agg.1) or aggregator 482 2 (Agg.2). The number of the EVs operated by Agg.1 and Agg.2 483 


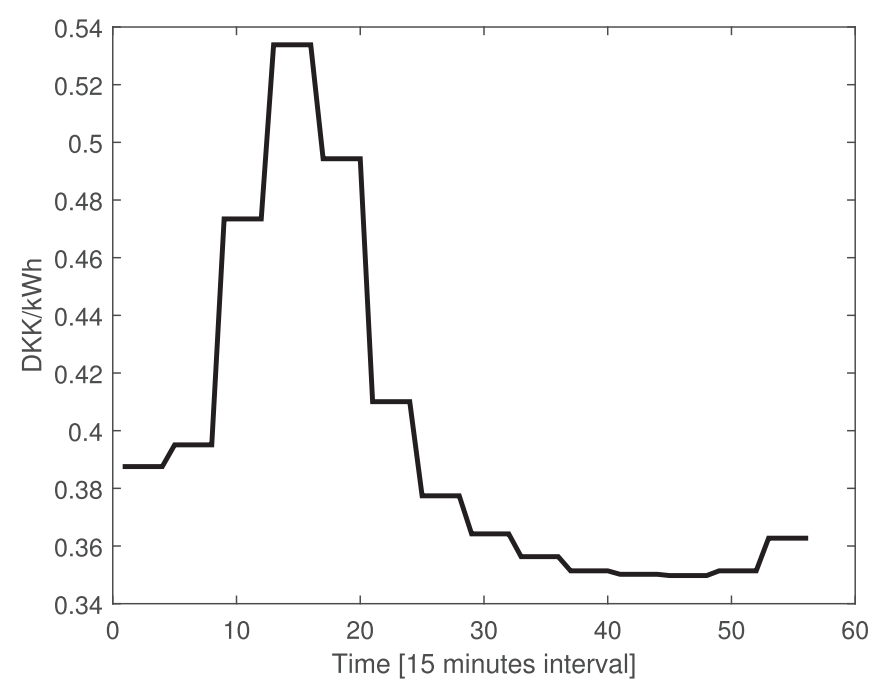

Fig. 3. Electricity energy price, an example from NordPool.

is 18 and 36 in each level, respectively. The scheduling period considered in this case is from 16.00 to 06.00 and a $15-\mathrm{min}$ interval is used. The hourly predicted day-ahead market price from 16.00 to 06.00 is assumed to be known to the aggregator and the price ${ }^{2}$ is shown in Fig. 3, the price will be used in stage I for generating EV charging schedule.

For other parameters in EV charging:

1) Battery capacity $E_{\text {cap }}$ is set to $24 \mathrm{kWh}$

2) $S O C_{o}$ is set to 0.2 of the battery capacity

3) $S O C_{\max }$ is set to $100 \%$ of the battery capacity

4) Maximum charging power is limited to $3.7 \mathrm{~kW}$ which fits with the Danish case (16 A, $230 \mathrm{~V}$ connection).

2) Distribution network and control parameters: A representative Danish distribution grid is illustrated in Fig. 4 where 72 households are connected to the feeders: 51 households are attached to the left branch and 21 households are located on the right side of the network. For the parameters used in the network-constrained transactive control, a time series base load is assumed to be known by the distribution system operators. With the base load, the DSO can calculate the base voltage, i.e., the $U_{0}$ in (4) per bus. In all time slots, the power transformer capacity allocated to two EV aggregators is $120 \mathrm{~kW}$ in both EV penetration cases, the minimum voltage $U_{\mathrm{Min}}$ per bus is assumed to be 0.905 p.u. for the $50 \% \mathrm{EV}$ penetration case and 0.88 p.u. for the $100 \% \mathrm{EV}$ penetration case. Note the 0.905 p.u. and 0.88 p.u. are given empirically, for the $100 \% \mathrm{EV}$ penetration case, the EV charging power is very high for the distribution network, but the method still converges for the relaxed voltage constraint. In reality, the minimal voltage 0.88 p.u. is not recommended, here it is mainly used for presenting the effectiveness of the proposed control method, even under the $100 \% \mathrm{EV}$ penetration case. The initial Lagrangian multipliers are assumed to be zero per bus in all the time slots and are updated per iteration to the aggregators and the DSO. The weighting factor rate $C_{1, i, l}$ and $C_{2, i, l}$ is set to 0.5 and 0.1 , respectively. A constant stepsize $\left(\alpha_{\omega}=0.1\right)$ is

${ }^{2}$ The electricity price assumed here is drawn from the real electricity price from NordPool spot market (http://www.nordpoolspot.com/)

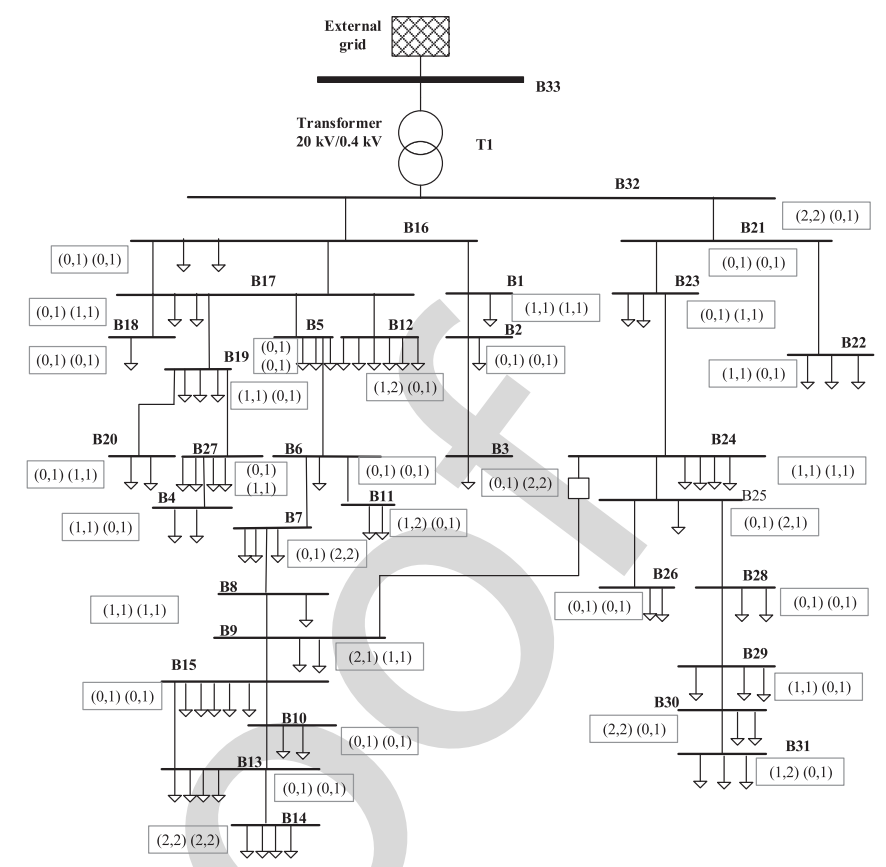

Fig. 4. A representative Danish distribution network with EV connected. We use two sets of parentheses inside the block under each bus index to show the EVs that are connected to the bus. The left set of parentheses represents Agg.1's EV information and the right one shows Agg.2's EV information. In each set of parentheses, the number of the EVs assigned to the two EV penetration levels is indicated (left for $50 \% \mathrm{EV}$ penetration case, right for $100 \% \mathrm{EV}$ penetration case).

chosen for the Lagrangian multiplier update. The value of $a$ and 519 $b$ is 0.1 and 300, respectively. 520

Note the values of $a$ and $b$ can influence the performance of 521 both DSO and aggregators. Therefore, the values must be tuned 522 properly when use in real. Technically, the value of $a$ and $b$ is 523 chosen based on empirical study in this work and the principle is 524 to make the optimum of different actors (DSO and aggregators) 525 have the same order of magnitude. Economically, the values 526 should be agreed based on negotiation between the DSO and 527 the aggregators, since it will influence the cost of aggregators 528 and DSO. It is noted there is work remaining on this matter, and 529 how exactly the process should be will be investigated in further 530 research effort.

\section{B. Simulation Scenarios}

With the provided parameters of the EVs, Agg.1 and Agg.2 533 calculate their optimal schedules according to (1). The power 534 schedule of the EVs is firstly allocated in the time period 45 to 48535 because of the lower electricity price, i.e., 02:00 to 03:00 AM, 536 thus this hour is used for illustrating the control performance. 537 The sum of the power in these time periods is higher than the 538 allocated power transformer's capacity. To illustrate the effec- 539 tiveness of the network-constrained transactive control and to 540 examine the effect of adding power loss objective function as 541 well as voltage constraints in (4), three scenarios are considered 542 here:

1) Scenario 1: Basic network-constrained transactive control. In this scenario, only congestion is considered, the
20

1

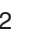

3

24

25

25
27

8

29

1

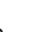

33

534

535

536

537

38

39

540

541
542



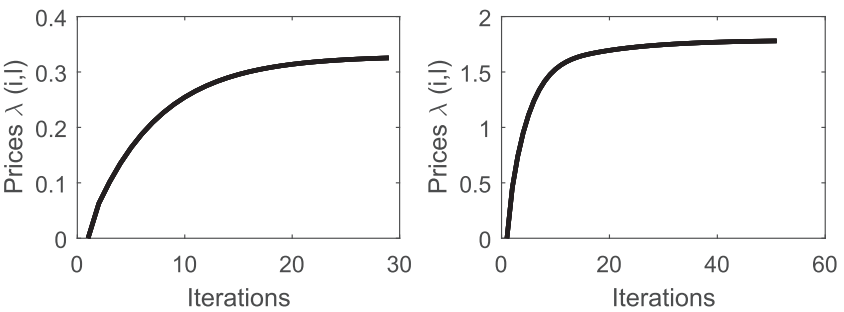

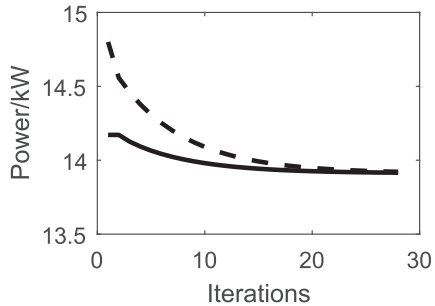

(a)

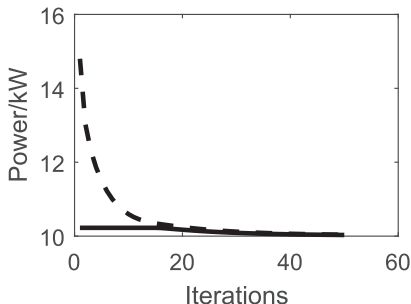

(b)
Fig. 5. Convergence of $\lambda(i, l)$ and power of DSO and aggregators at bus 14 $i=45, \ldots 48$, in scenario 1. Dotted power profile: The sum of Agg.1 and Agg.2; solid power profile: DSO.

power loss and the voltage constraints are not included in the optimization problems.

2) Scenario 2: Network-constrained transactive control with voltage constraints. In this scenario, the voltage constraints are included on top of scenario 1.

3) Scenario 3: Network-constrained transactive control with voltage constraints and power loss. In this scenario, the power loss objective is included on top of scenario 2 .

Note the method does not require a fixed bus location of individual EV; however, in order to compare the differences between these scenarios, we use the same setting for electric vehicles' locations in the network that is shown in Fig. 4.

\section{Simulation Results}

1) Scenario 1: Fig. 5(a) shows the simulation result of the $50 \% \mathrm{EV}$ penetration case where the problem is solved after 29 iterations. It means the DSO and the aggregators reach consensus in terms of power at each bus for all the time slots. The power of the DSO and aggregators is regulated by the shadow prices presented in the upper level of the figure. In the simulation, bus 14 has the lowest voltage and thus the power profile of DSO and aggregators at bus 14 is presented. The figure shows that four electric vehicles are initially scheduled to charge from 02:00 to 03:00 AM. However, to respect the power transformer constraint, the charging power is reduced in this hour and the required additional energy is compensated in other time slots that is not shown here. To demonstrate the changes before and after the control, the charging profile of EVs on bus 14 (including two EVs of Agg. 1 and two EVs of Agg.2) is shown in Fig. 6 during the entire scheduling period. In addition, Fig. 5(b) shows the results of the $100 \% \mathrm{EV}$ penetration case. The congestion price increases in this case because of the higher EV charging power, correspondingly, the converged power of the DSO and the aggregators is less than the one in $50 \% \mathrm{EV}$ penetration case.

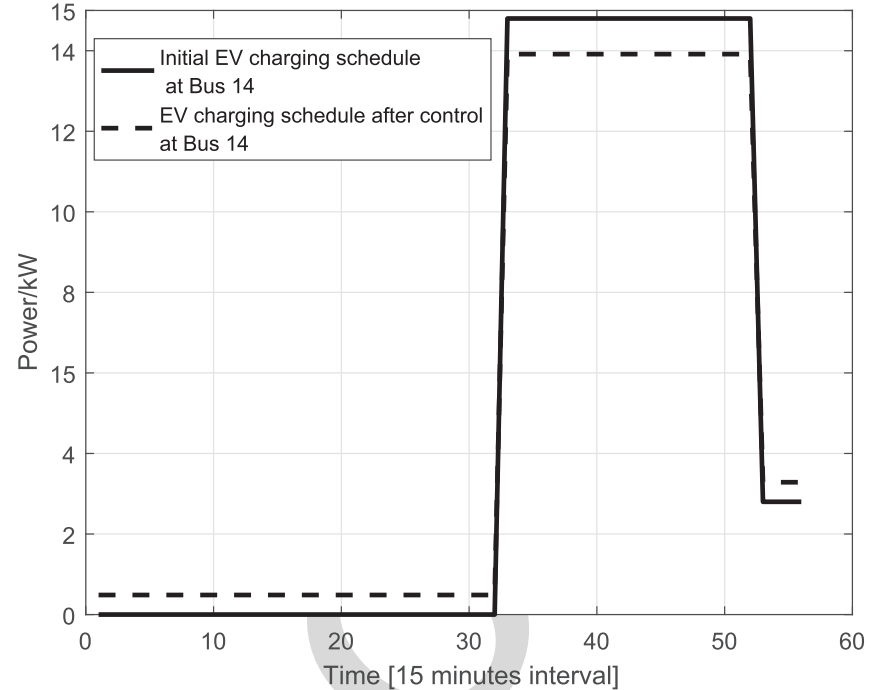

Fig. 6. Comparison of the charging schedule of EVs connected at bus 14 in presence of control in scenario 1 .
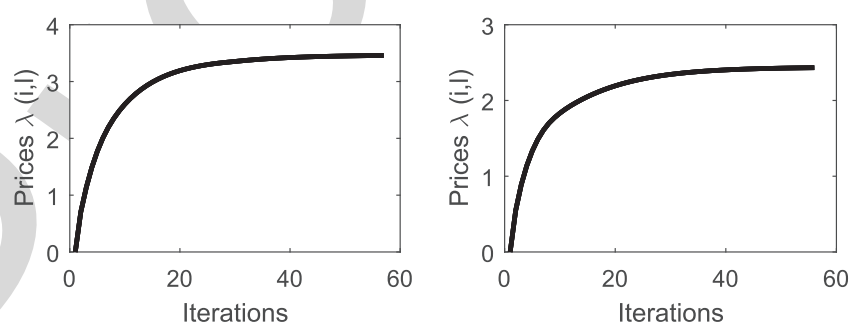

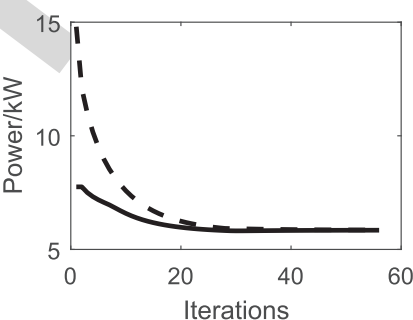

(a)

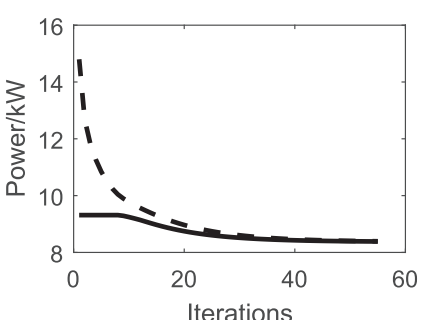

(b)
Fig. 7. Convergence of $\lambda(i, l)$ and power of DSO and aggregators at bus 14, $i=45, \ldots 48$, in scenario 2. Dotted power profile: the sum of Agg.1 and Agg.2; solid power profile: DSO.

2) Scenario 2: In this scenario, bus voltage constraints are 579 included in the optimization problem. Fig. 7(a) presents the con- 580 vergence of the power and the congestion price. Compared with 581 Fig. 5(a), the results indicate longer iterations are needed to 582 reach the convergence. Besides, the congestion prices increase 583 a lot to further reduce the power at bus 14 during these four 584 time periods (i.e., 45 to 48 ) and the purpose is to ensure that the 585 voltage is not violated. Table I presents the voltage comparison 586 calculated from scenario 1 and scenario 2 . In each scenario, we 587 calculate the voltage using the loading profiles (base load plus 588 the EVs charging load) before and after the transactive control. It 589 can be seen that the minimum voltage of the distribution network 590 in scenario 2 increases a lot compared with the one in scenario 1, 591 which show the effectiveness of the voltage approximation 592 method in (5) and (6). The minimum voltage is recalculated 593 
TABLE I

Power Losses And Voltage Before and After Transactive Control

\begin{tabular}{llcccc}
\hline \hline \multicolumn{6}{c}{ Electric Vehicle With 50\% Penetration } \\
\hline Scenarios & Control & Loss (MWh) & Energy (MWh) & Loss ratio & Voltage (p.u.) \\
\hline \multirow{2}{*}{ Scenario 1 } & Before control & 0.1348 & 2.0699 & $6.51 \%$ & 0.8548 \\
& After control & 0.1270 & 2.0611 & $6.16 \%$ & 0.8634 \\
Scenario 2 2 & Before control & 0.1348 & 2.0699 & $6.51 \%$ & 0.8548 \\
& After control & 0.1106 & 2.0454 & $5.41 \%$ & $\mathbf{0 . 9 0 3 5}$ \\
Scenario 3 3 & Before control & 0.1348 & 2.0699 & $6.51 \%$ & 0.8548 \\
& After control & 0.1096 & 2.0443 & $5.36 \%$ & $\mathbf{0 . 9 0 3 6}$ \\
\hline \multicolumn{6}{c}{ Electric Vehicle With 100\% Penetration } \\
\hline Scenarios & Control & Loss (MWh) & Energy (MWh) & Loss ratio & Voltage (p.u.) \\
\hline Scenario 1 & Before control & 0.3086 & 2.9349 & $10.51 \%$ & 0.7675 \\
& After control & 0.1904 & 2.8150 & $6.76 \%$ & 0.8684 \\
Scenario 2 & Before control & 0.3086 & 2.9349 & $10.51 \%$ & 0.7675 \\
& After control & 0.1893 & 2.8148 & $6.72 \%$ & 0.8753 \\
Scenario 3 3 Before control & 0.3086 & 2.9349 & $10.51 \%$ & 0.7675 \\
& After control & 0.1890 & 2.8150 & $6.71 \%$ & 0.8753 \\
\hline \hline
\end{tabular}

after the power reaches consensus and thus the voltage is not exactly the expected 0.905 p.u. in all scenarios. We note that, compared with scenario 1 , the voltage profiles in scenario 2 are kept above 0.9 p.u. that fulfills the European standard EN 50160. The voltage results of the $100 \% \mathrm{EV}$ penetration case are also presented, the voltage here illustrates the effectiveness of the method, since compared to scenario 1 of $100 \%$ EV penetration case, the voltage increases. In addition, Fig. 7(b) shows the results of the $100 \% \mathrm{EV}$ penetration case. The congestion price increases a bit in this case compared with the one in Fig. 5(b) because of the voltage constraints.

3) Scenario 3: Compared with scenario 2, the power loss objective is included in the optimization problem. Similarly, the power of the DSO and the aggregators as well as the regulating congestion prices during the transactive control are shown in Fig. 8(a). The results indicate that a longer iteration number is required before consensus is reached. Besides, the congestion price is higher and thus the converged power is smaller than the one shown in Fig. 7(a). Furthermore, we compare the power loss of scenario 3 with scenarios 1 and 2 . The results are shown in Table I. Here, the power loss ratio is a relationship between the energy losses and the energy injected at bus 33 . The results show that the loss in scenario 3 is optimal compared with the one in scenario 2 . The minimum voltage of scenario 3 is also included in Table. I. In addition, Fig. 8(b) shows the results of the $100 \% \mathrm{EV}$ penetration case. The congestion price increases further in this case compared with the one in Fig. 7(b) because of the inclusion of objective loss.

\section{Optimality Verification}

To investigate the optimality of the numerical result obtained with the network-constrained transactive control method, we compare the results with the one solved directly from the optimization problem (7) that is named centralized control. Table II presents the results obtained in each scenario for the two EV penetration levels. The value shown in the table is the power at bus 14 corresponding to time slot 45 . It is seen that the value obtained by centralized control (Central) and transactive control
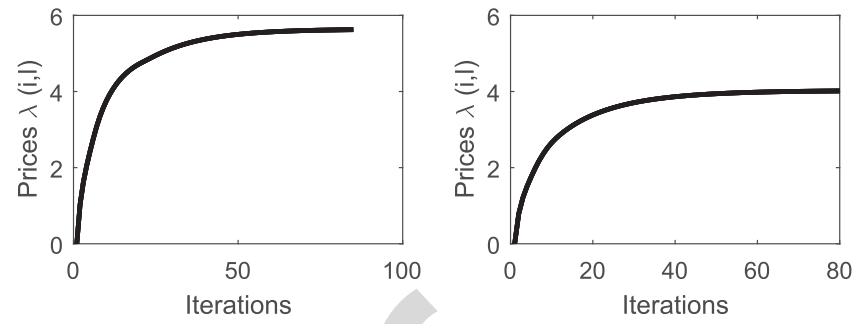

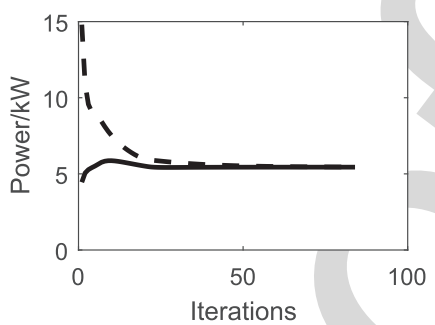

(a)

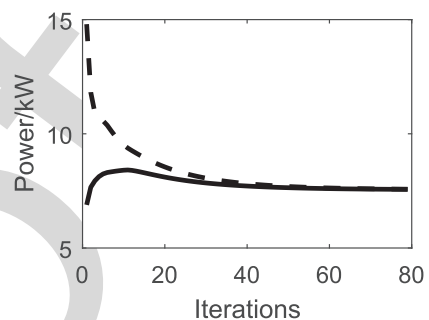

(b)
Fig. 8. Convergence of $\lambda(i, l)$ and power of DSO and aggregators at bus 14 $i=45, \ldots 48$, in scenario 3. Dotted power profile: The sum of Agg.1 and Agg.2; solid power profile: DSO.

TABLE II

COMPARISON OF SCENARIOS SOLVED BY CENTRALIZED CONTROL AND TRANSACTIVE CONTROL

\begin{tabular}{|c|c|c|c|c|c|}
\hline \multicolumn{2}{|c|}{ EV Penetrations } & \multicolumn{2}{|c|}{$50 \%$ Penetration } & \multicolumn{2}{|c|}{$100 \%$ Penetration } \\
\hline & & $P_{\mathrm{DSO}}$ & $P_{\mathrm{Agg}}$ & $P_{\mathrm{DSO}}$ & $P_{\mathrm{Agg}}$ \\
\hline \multirow[t]{2}{*}{ Scenario 1} & Central & 13.9118 & 13.9118 & 10.0159 & 10.0159 \\
\hline & Transactive & 13.9156 & 13.9218 & 10.0312 & 10.0380 \\
\hline \multirow[t]{2}{*}{ Scenario 2} & Central & 5.8490 & 5.8490 & 8.3725 & 8.3725 \\
\hline & Transactive & 5.8457 & 5.8549 & 8.3854 & 8.3935 \\
\hline \multirow[t]{2}{*}{ Scenario 3} & Central & 5.3661 & 5.3661 & 7.5809 & 7.5809 \\
\hline & Transactive & 5.4410 & 5.4507 & 7.5662 & 7.5747 \\
\hline
\end{tabular}

(Transactive) is comparable, which verifies the optimality of the 631 proposed model. Note from algorithm perspective, the proposed 632 method is solved by introducing a Lagrange multiplier $\lambda$ and the 633 dual problem gives the same solution as the one in centralized 634 control due to the convexity of the optimization problem [24]. 635 Thus we concludes the optimality of the proposed method with 636 the comparison, although the solution of the central and trans- 637 active control in the table is not exactly the same because the 638 problem is solved numerically here.

\section{Discussion AND CONCLUSIONS}

In this study, the bid cost function that EV aggregators used to express their charging flexibility to the price coordinator is quadratic, as discussed in [16], popular utility/cost functions include a linear and quadratic utility function which means equilibrium prices can usually be found. However, in some situations, the equilibrium may not be identified. In this case, relaxation of the constraints or heuristic methods may be needed. Furthermore, note that the case study is towards EU system where the distribution network is normally planned as three phases, also the approximation of the load flow model though is not exact however the results show the effectiveness. As application of this method to unbalanced distribution system, it
641 642 643 644 645 646 647 648 649 650 651 652 
is applicable and in that case the adaption requires introducing lamada, i.e., the shadow price on each phase.

In addition, it is one of the assumptions that there are flexibilities within an EV fleet who can shift the demand over a planning horizon to avoid high market price. For a few inflexible customers, their demands can be handled in the aggregators optimisation model by adding additional constraints for their specific energy charging requirements. If it causes violations of network constraints or higher charging cost, then there should be mechanisms between the aggregators and the customers to handle such issue.

Although the EV is used as an example to illustrate the effectiveness of the proposed method, it is note that the method can also be extended to capture other flexible loads such as heat pumps and storages. In addition, the model can be also demonstrated in a distribution system with high penetration of distributed generator such as wind/solar generators. Under this circumstance, the condition will become complex, such as the distributed generator might bring over-voltage problem, if it is the case, a similar penalized method could be used to manage the power flow of the distributed generators. Moreover, it is envisioned that, if distributed generations have contracts with the aggregator, the distributed generator and the flexible loads should be jointly optimally operated by the aggregator, then the DSO only interacts with the aggregators based on the net-power (generation minus consumption) of the aggregator.

To sum up, this paper develops a network-constrained transactive control method and applies it specifically for integrating electric vehicles into power distribution systems. The proposed modeling method covers multiple time periods, which extends the application of transactive control that has been reported in previous studies. The extensions make the transactive control technique fit better with the normal operation of power system operators since 'schedule and control' is a typical approach used by the system operators. Furthermore, the proposed method considers the energy inter-temporal characteristics of electric vehicles, i.e., the dynamics of electric vehicle charging. By using the proposed transactive control method, the system operator can ensure a safe operation of the network and the aggregators can optimize the electric vehicles' charging schedules.

The merit of the work is that it represents a decentralized operation instead of a centralized dispatch, as for centralized mechanism, there would be questions like computational requirements issue, privacy issue? Such questions are addressed and eliminated through transactive control, as each actors keep their operational cost functions and only communicate the solutions with the price coordinator through a negotiation mechanism.

1] D. Apostolopoulou, S. Bahramirad, and A. Khodaei, "The interface of power: Moving toward distribution system operators," IEEE Power Energy Mag., vol. 14, no. 3, pp. 46-51, May/Jun. 2016.

[2] S. D. Manshadi and M. E. Khodayar, "A hierarchical electricity market structure for the smart grid paradigm," IEEE Trans. Smart Grid, vol. 7, no. 4, pp. 1866-1875, Jul. 2016.

[3] C. Cecati, C. Citro, and P. Siano, "Combined operations of renewable energy systems and responsive demand in a smart grid," IEEE Trans. Sustain. Energy, vol. 2, no. 4, pp. 468-476, Oct. 2011.
[4] D. T. Nguyen, H. T. Nguyen, and L. B. Le, "Dynamic pricing design for 711 demand response integration in power distribution networks," IEEE Trans. 712 Power Syst., vol. 31, no. 5, pp. 3457-3472, Sep. 2016.

[5] R. B. Melton, "Gridwise transactive energy framework version 1," GridWise Archit. Council, Richland, WA, USA, Tech. Rep. PNNL-22946, 2015 .

[6] A. Nilgun and Z. Marzia, "Transactive energy: A surreal vision or a necessary and feasible solution to grid problems?," California Public Utilities Commission, Policy Planning Division, San Francisco, CA, USA, Tech. Rep., 2014.

[7] K. Kok, C. Warmer, and I. Kamphuis, "Powermatcher: Multiagent control in the electricity infrastructure," in Proc. 4th ACM Int. Joint Conf. Auton. Agents Multiagent Syst., New York, NY, USA, 2005, pp. 75-82.

[8] A. K. Bejestani, A. Annaswamy, and T. Samad, "A hierarchical transactive control architecture for renewables integration in smart grids: Analytical modeling and stability," IEEE Trans. Smart Grid, vol. 5, no. 4, pp. 2054-2065, Jul. 2014.

[9] D. J. Shiltz, M. Cvetkovi, and A. M. Annaswamy, "An integrated dynamic market mechanism for real-time markets and frequency regulation," IEEE Trans. Sustain. Energy, vol. 7, no. 2, pp. 875-885, Apr. 2016.

[10] S. Li, W. Zhang, J. Lian, and K. Kalsi, "Market-based coordination of thermostatically controlled loads-part I: A mechanism design formulation," IEEE Trans. Power Syst., vol. 31, no. 2, pp. 1170-1178, Mar. 2016.

[11] S. Vandael, B. Claessens, M. Hommelberg, T. Holvoet, and G. Deconinck, "A scalable three-step approach for demand side management of plug-in hybrid vehicles," IEEE Trans. Smart Grid, vol. 4, no. 2, pp. 720-728, Jun. 2013. driven dual coordination mechanism for demand side management of 739 phevs," IEEE Trans. Smart Grid, vol. 5, no. 2, pp. 751-760, Feb. 2014.

[13] Z. Darabi and M. Ferdowsi, "Aggregated impact of plug-in hybrid electric 741 vehicles on electricity demand profile," IEEE Trans. Sustain. Energy, 742 vol. 2, no. 4, pp. 501-508, Oct. 2011

[14] M. S. ElNozahy and M. M. A. Salama "A comprehensive study of the impacts of PHEVs on residential distribution networks," IEEE Trans. 745 Sustain. Energy, vol. 5, no. 1, pp. 332-342, Jan. 2014.

[15] Y. O. Assolami and W. G. Morsi, "Impact of second-genertion plugin battery electric vehicles on the aging of distribution transformers 748 considering TOU prices," IEEE Trans. Sustain. Energy, vol. 6, no. 4, pp. $1606-1614$, Oct. 2015

[16] S. Weckx, R. D'hulst, B. Cl ing of electric vehicles respecting distribution transformer loading and voltage limits," IEEE Trans. Smart Grid, vol. 5, no. 6, pp. 2857-2867, Nov. 2014.

[17] S. Huang, Q. Wu, S. S. Oren, R. Li, and Z Liu "Distribution locational marginal pricing through quadratic programming for congestion manage- 756 ment in distribution networks," IEEE Trans. Power Syst., vol. 30, no. 4, 757 pp. 2170-2178, Jul. 2015.

[18] H. Chao, "Demand response in wholesale electricity markets: The choice 759 of customer baseline," J. Regulatory Econ., vol. 39, no. 1, pp. 68-88, 760 Nov. 2011.

[19] O. Sundstrom and C. Binding, "Flexible charging optimization for electric vehicles considering distribution grid constraints," IEEE Trans. Smart Grid, vol. 3, no. 1, pp. 26-37, Mar. 2012.

[20] F. Marra, G. Yang, C. Træholt, J. Østergaard, and E. Larsen, "A decentralized storage strategy for residential feeders with photovoltaics," IEEE Trans. Smart Grid, vol. 5, no. 2, pp. 974-81, Mar. 2014

[21] G. Yang et al., "Voltage rise mitigation for solar PV integration at LV grids," J. Modern Power Syst. Clean Energy, vol. 3, no. 3, pp. 411-21, Sep. 2015.

[22] N. Gatsis and G. Giannakis, "Residential load control: Distributed scheduling and convergence with lost AMI messages," IEEE Trans. Smart Grid, vol. 3, no. 2, pp. 770-786, Jun. 2012.

[23] B. Moradzadeh and K. Tomsovic, "Two-stage residential energy management considering network operational constraints," IEEE Trans. Smart Grid, vol. 4, no. 2, pp. 2339-2346, Dec. 2013.

[24] S. Boyd, L. Xiao, and A. Mutapcic, "Subgradient methods," Stanford University, Stanford, CA, USA, Autumn Quarter, 2003.

[25] M. Grant and S. Boyd, CVX: Matlab software for disciplined convex programming, version 2.1, (Mar. 2014). [Online]. Available: http://cvxr. $\mathrm{com} / \mathrm{cvx}$

[26] M. Grant and S. Boyd, "Graph implementations for nonsmooth convex programs," in Recent Advances in Learning and Control. Berlin, Germnay: Springer, 2008, pp. 95-110. Available: http://stanford.edu/ boyd/graph_dcp.html 730 731
732 733 748 749

\section{,}

16 729 734 35 36
37 . 40 43 44
45 46 


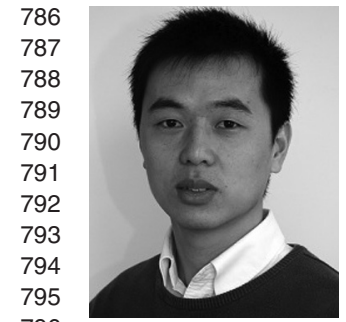

Junjie Hu (M'xx) received the M.Sc. degree in control theory and control engineering from TongJi University, Shanghai, China, in 2010, and the Ph.D. degree in electrical engineering from Technical University of Denmark, Kongens Lyngby, Denmark, in 2014. He is currently a Postdoctoral Researcher with the Department of Electrical Engineering, Technical University of Denmark. His main research interests include distributed energy resources integration with focus on electric vehicles, application of optimal control theory on active distribution grid operation and 797
798 University of Denmark. Since 2009, he has been leading several industrial collaborative projects in Denmark in the field of monitoring, operation and protection of renewable energy systems. His research interests include renewable energy integration, smart grids, and cyber physical energy systems.

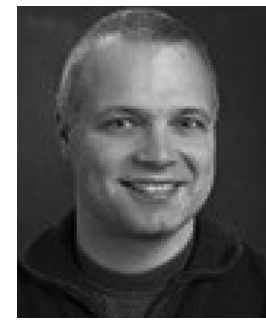

Henrik W. Bindner (M'xx) received the M.Sc. degree in electrical engineering from the Technica University of Denmark, Lyngby, Denmark, in 1988. Since 1990, he has been with the Ris National Laboratory for Sustainable Energy, Roskilde, Denmark, in the Wind Energy Division. Since 2008, he has been a Senior Scientist with the Department for Electrical Engineering, Ris Campus, Technical University of Denmark. His main research interests include integration of wind energy into power systems, as well as analysis, design, and control of small island systems.
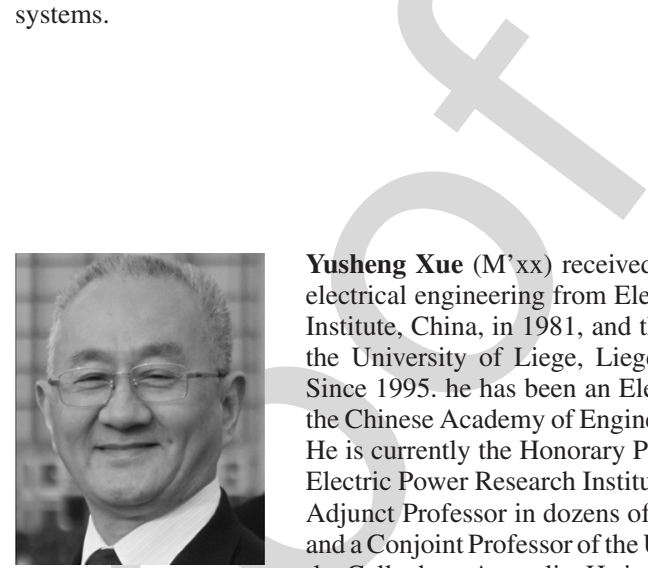

Yusheng Xue (M'xx) received the M.Sc. degree in 826 electrical engineering from Electric Power Research Institute, China, in 1981, and the Ph.D. degree from the University of Liege, Liege, Belgium, in 1987. Since 1995. he has been an Elected Academician of the Chinese Academy of Engineering Beijing, China. $\mathrm{He}$ is currently the Honorary President of State Grid Electric Power Research Institute, Beijing, China, an Adjunct Professor in dozens of universities in China and a Conjoint Professor of the University of Newcastle, Callaghan, Australia. He is the Editor-in-Chief of Automation of Electric Power System (in Chinese) and that of Journal of Modern Power Systems and Clean Energy (in English), as well as Chairman of the Technical Committee of Chinese National Committee of CIGRE, since 2005.
813 
Q1. Author: Authors, when accessing and uploading your corrections at the Author Gateway, please note we cannot accept new source files as corrections for your paper. Do not send new Latex, Word, or PDF files, as we cannot simply "overwrite" your paper. Please submit your corrections as an annotated PDF or as clearly written list of corrections, with location in paper. You can also upload revised graphics to the Author Gateway.

Q2. Author: If you need an invoice or have any other billing questions, please contact reprints@ieee.org as they handle these requests.

Q3. Author: Please provide technical report number in Ref. [6].

Q4. Author: Please provide full bibliographic details in Ref. [24].

Q5. Author: Please provide IEEE membership year of all the authors. 